\title{
Effect of intraoperative dexmedetomidine infusion on delirium in adult patients following cardiac valve surgery: a protocol of a randomized, double-blinded, and placebo-controlled study
}

\author{
Hong-Bai Wang ${ }^{1+}$, Liang Zhang ${ }^{2 \dagger}$, Zhe Zhang ${ }^{1}$, Su Yuan ${ }^{1 *} \mathbb{D}$, Fu-Xia Yan ${ }^{1}$ and Qi-Peng Luo ${ }^{1}$
}

\begin{abstract}
Background: Delirium is an acute status of brain dysfunction that commonly occurs in patients who have undergone cardiac surgery, and increases morbidity and mortality. It is associated with risk factors, such as older age, use of narcotics, cardiopulmonary bypass, and hypothermia. Dexmedetomidine infusion might exert a neuroprotective effect. However, the effect of perioperative administration of dexmedetomidine on the incidence of postoperative delirium (POD) in patients undergoing cardiac or non-cardiac surgery is yet controversial. The present study aimed to reveal the effect of intraoperative dexmedetomidine administration on the incidence of delirium in adult patients following cardiac surgery.
\end{abstract}

Methods: This single-center, randomized, double-blinded, and placebo-controlled trial consisted of 652 patients randomly divided into two groups: dexmedetomidine and placebo. $0.6 \mu \mathrm{g} / \mathrm{kg}$ dexmedetomidine will be infused $10 \mathrm{~min}$ after central vein catheterization, followed by a continuous infusion at a speed of $0.4 \mu \mathrm{g} / \mathrm{kg} / \mathrm{h}$ until the end of surgery in the dexmedetomidine group, while normal saline will be administered at the same rate in the placebo group. The primary outcome is the incidence of POD during the first 7 days post-surgery. The secondary outcomes include duration of mechanical ventilation after surgery, duration of stay in the intensive care unit and the hospital after surgery, incidence of hypotension during or after dexmedetomidine infusion, acute kidney injury and sudden arrhythmia during the hospital stay postoperatively, and all-cause mortality in 30 and 90 days after surgery, respectively.

Discussion: This study was approved by the Ethics Committee of the Chinese Academy of Medical Sciences Fuwai Hospital on 6 March 2019 (2019-1180). The results will be disseminated at academic conferences and submitted to peer-reviewed publications. Either positive or negative results will provide guidance for clinical practice.

\footnotetext{
*Correspondence: fuwaiys@126.com

${ }^{\dagger}$ Hong-Bai Wang and Liang Zhang contributed equally and should be regarded as co-first authors.

'Department of Anesthesiology, Fuwai Hospital, Chinese Academy of Medical Sciences and Peking Union Medical College, Beijing, No. 167 North Lishi Road, Xicheng District, Beijing, China

Full list of author information is available at the end of the article
}

C C The Author(s). 2020 Open Access This article is licensed under a Creative Commons Attribution 4.0 International License, which permits use, sharing, adaptation, distribution and reproduction in any medium or format, as long as you give appropriate credit to the original author(s) and the source, provide a link to the Creative Commons licence, and indicate if changes were made. The images or other third party material in this article are included in the article's Creative Commons licence, unless indicated otherwise in a credit line to the material. If material is not included in the article's Creative Commons licence and your intended use is not permitted by statutory regulation or exceeds the permitted use, you will need to obtain permission directly from the copyright holder. To view a copy of this licence, visit http://creativecommons.org/licenses/by/4.0/. The Creative Commons Public Domain Dedication waiver (http://creativecommons.org/publicdomain/zero/1.0/) applies to the data made available in this article, unless otherwise stated in a credit line to the data. 
(Continued from previous page)

Trial registration: The Chinese Clinical Trial Registry (http://www.chictr.org.cn) ChiCTR1900022583. Registered on 17 April 2019.

Keywords: Dexmedetomidine, Cardiac valve surgery, Delirium, Adult

\section{Strengths and limitations of the study Strengths}

(1) This study includes the largest sample size of all studies with respect to the effect of dexmedetomidine on postoperative delirium in patients undergoing cardiac surgeries.

(2) The Chinese Academy of Medical Sciences Fuwai Hospital is a world-class center of cardiac surgery and surgeons and anesthetists with extensive experience in the perioperative management of patients undergoing cardiac surgery.

(3) We will select only the patients undergoing cardiac valve surgery to decrease the bias due to a discrepancy in the surgery types.

(4) All investigators who will perform the follow-up will have undergone training by a senior psychiatrist about the diagnosis of delirium.

(5) The long-term endpoints will be observed.

\section{Limitations}

(1) As it is a single-center study, the dissemination of the results may be limited.

(2) As some delirium patients may be missed at the follow-up time, a higher incidence of delirium might occur.

(3) The Richmond Agitation-Sedation Scale and the Confusion Assessment Method for Intensive Care Unit can produce measurement bias because the two questionnaires are subjective.

(4) Lack of interim analysis can lead to an inaccurate sample size, although the sample size in this study is the largest.

\section{Background}

Delirium is a type of brain dysfunction with an acute onset and fluctuating occurrence [1]. According to several clinical studies, the patients have a high prevalence of delirium after cardiac surgery, which varies from 5 to $72 \%[2,3]$. Several predisposing factors are considered to be associated with the development of postoperative delirium (POD) in patients following cardiac surgery, such as the patients with age $\geq 65$ years old [4], narcotics [5], cardiopulmonary bypass (CPB) [6], perioperative poor sleep quality [7], and enhanced postoperative pain sensitivity [8]. POD significantly elevates the incidence of adverse events and 30-day mortality [9-11].

Dexmedetomidine is a medication with a high affinity for $\alpha_{2}$ adrenergic receptor, leading to a decrease in noradrenaline release by activating the $\alpha_{2}$ adrenergic receptors in the central nervous system (CNS), initiating sedation [12]. In addition, dexmedetomidine can be used as a medication for anti-anxiety, improvement of sleep quality, and analgesia [13]. Currently, the drug is widely used in clinical anesthesia and intensive care units (ICU) as an adjuvant sedative.

Interestingly, dexmedetomidine might decrease the incidence of delirium in patients with high risk. A large number of prospective studies about the effect of prophylactic dexmedetomidine on delirium have been published, although most of them included patients undergoing non-cardiac surgeries or were in ICU without surgical procedures [14-21]. Relatively fewer prospective studies have been carried out on heart surgeries, and the majority of these studies adopted the method of perioperative medication [22-24]. Some studies reported intraoperative administration of dexmedetomidine vs. placebo $[25,26]$. A prospective study by Shu et al. [25] found that intraoperative infusion of dexmedetomidine significantly decreased the incidence of POD in patients undergoing cardiac surgery as compared to placebo. However, this study only included 60 cases, and the results had poor reliability. Another study with a larger sample size and longer infusion time of dexmedetomidine demonstrated a negative result with respect to the incidence of POD as compared to placebo [23]. Hence, a prospective randomized controlled trial (RCT) with a large-scale sample size is required to further prove the effect of intraoperative dexmedetomidine infusion on POD in patients undergoing cardiac surgery. In this prospective, double-blinded, randomized, placebocontrolled study with a large sample size, we expect to reveal the effect of the intraoperative prophylactic administration of dexmedetomidine at a routine rate on the incidence of POD in patients undergoing cardiac surgery.

\section{Methods}

Study design

This prospective, double-blinded, randomized, placebocontrolled trial will observe the superiority of dexmedetomidine infusion during cardiac surgery on delirium 
incidence in the first 7 days after the surgery. The entire progress of trial adheres to the principles of the World Medical Association's Declaration of Helsinki. An internal Data and Safety Monitoring Board (DSMB) has been constructed to supervise data collection and participants' safety and will decide on the continuation, modification, or cessation of the trial according to the National Institute of Health (NIH) guidelines. There is no financial and non-financial conflict of interests, as requested by the NIH regulation. The final study version 3.0 was approved by the Ethics Committee of the Chinese Academy of Medical Sciences, Fuwai Hospital, China, on 6 March 2019 (2019-1180). The study was registered in the Chinese Clinical Trial Registry on 17 April 2019 (identification number: ChiCTR1900022583). This protocol includes all components as described in the Standard Protocol Items: Recommendations for Interventional Trials (SPIRIT) checklist (Supplementary file 1) [27]. Figure 1 demonstrates the SPIRIT including the schedule of enrolment, interventions, and assessments of the trial. All eligible patients will be randomly divided into two groups at a ratio of 1:1: dexmedetomidine (DEX) and placebo (PLA). The screening of patients, intraoperative intervention, and postoperative delirium assessment will be performed at the Chinese
Academy of Medical Sciences, Fuwai Hospital. The flow chart of this study is shown in Fig. 2.

\section{Participants}

Adult patients (age $\geq 18$ years), who are scheduled to undergo open heart cardiac valve surgery on pump under general anesthesia, will be screened for inclusion in this study. Those patients who met either of the following criteria will be excluded: (1) refuse to provide written informed consent; (2) have a history of schizophrenia, epilepsy, Parkinson's disease, myasthenia gravis, or severe dementia; (3) cannot communicate due to visual, hearing, language, or other barriers; (4) have a history of brain trauma or neurosurgery; (5) have a left ventricular ejection fraction $<30 \%$; (6) have a history of sick sinus syndrome, severe bradycardia (a heart rate $<$ 50 beats/min), or an atrioventricular block of degree $\geq 2$; (7) have severe hepatic failure (Child-Pugh Grade C); and (8) have severe renal failure (requirement of renal replacement therapy).

\section{Patient recruitment and baseline data collection}

The investigators will screen the eligible patients the day before the surgery and explain the potential risks and benefits of the study protocol to the patients in person.

\begin{tabular}{|c|c|c|c|c|c|c|c|c|}
\hline \multirow[b]{3}{*}{ TIMEPOINT ${ }^{\star \star}$} & \multicolumn{8}{|c|}{ STUDY PERIOD } \\
\hline & \multirow{2}{*}{$\begin{array}{c}\text { Enrolment } \\
\text { Pre-op } \\
\text { period to } \\
\text { the day of } \\
\text { surgery }\end{array}$} & \multirow{2}{*}{$\begin{array}{c}\text { Allocation } \\
\begin{array}{c}\text { Day of } \\
\text { surgery }\end{array}\end{array}$} & \multicolumn{5}{|c|}{ Post-allocation } & \multirow{2}{*}{$\begin{array}{c}\text { Close-out } \\
\begin{array}{c}7 \text { days after } \\
\text { surgery }\end{array}\end{array}$} \\
\hline & & & $t_{1}$ & $t_{2}$ & $t_{3}$ & $t_{4}$ & $t_{5}$ & \\
\hline \multicolumn{9}{|l|}{ ENROLMENT: } \\
\hline Eligibility screen & $\mathrm{x}$ & & & & & & & \\
\hline Informed consent & $x$ & & & & & & & \\
\hline Allocation & & $\mathrm{x}$ & & & & & & \\
\hline \multicolumn{9}{|l|}{ INTERVENTIONS: } \\
\hline \multicolumn{9}{|l|}{ Dexmedetomidine } \\
\hline \multicolumn{9}{|l|}{ Placebo } \\
\hline \multicolumn{9}{|l|}{ ASSESSMENTS: } \\
\hline \multirow{3}{*}{$\begin{array}{c}\text { Baseline variables } \\
\text { Hemodynamic } \\
\text { parameters }\end{array}$} & $\mathrm{x}$ & $\mathrm{x}$ & & & & & & \\
\hline & & & $\mathrm{x}$ & $\mathrm{x}$ & $\mathrm{x}$ & $\mathrm{x}$ & $\mathrm{x}$ & $\mathrm{x}$ \\
\hline & & & & & & & & $X$ \\
\hline
\end{tabular}

Fig. 1 Standard Protocol Items: Recommendations for Interventional Trials (SPIRIT) figure. The schedule of enrollment, interventions, and assessments in the study. $t_{1}$, before anesthetic induction; $t_{2}$, after anesthetic induction; $t_{3}$, after central venous catheterization; $t_{4}$, during surgery; $t_{5}$, after surgery 


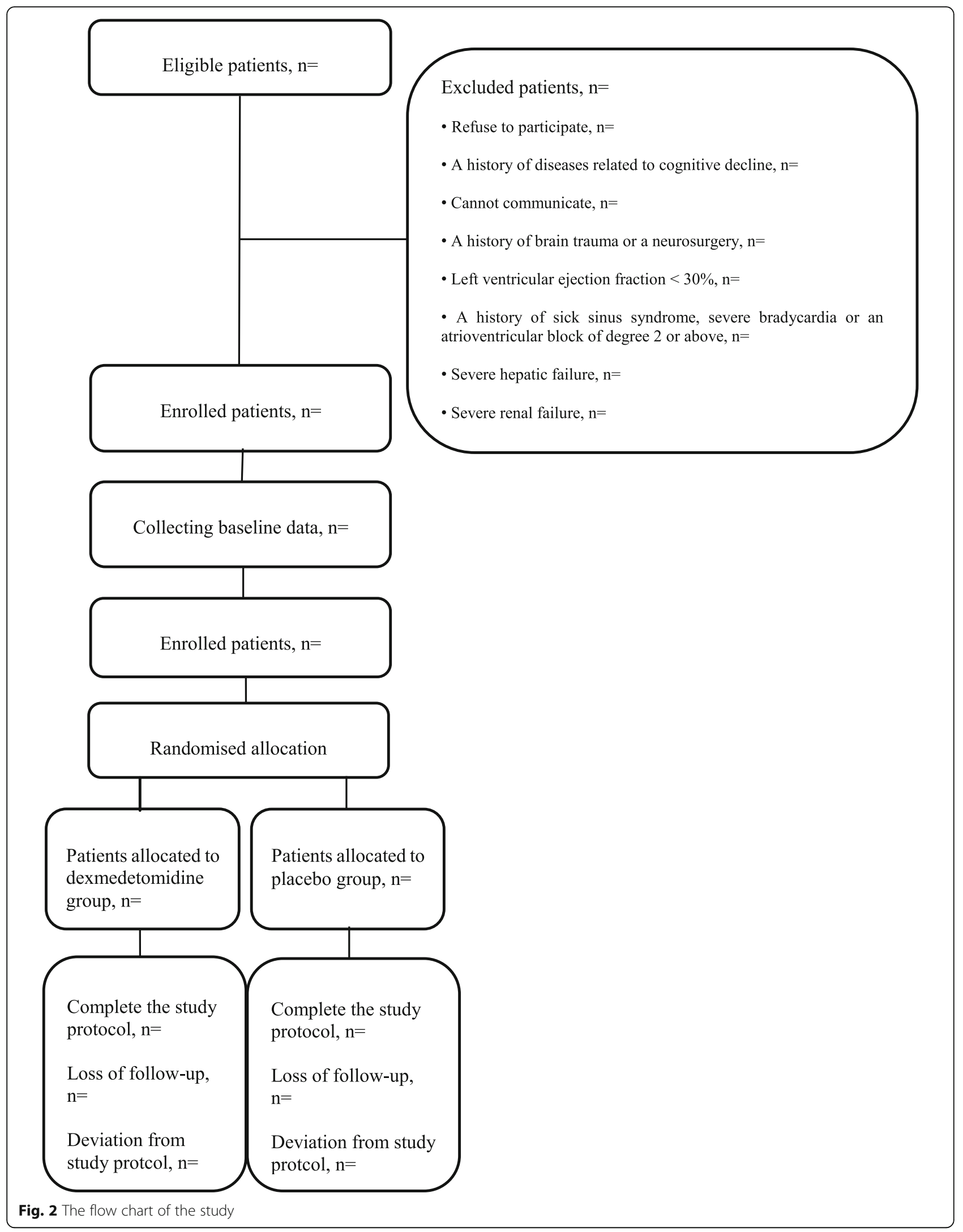


The patients who do not fulfill any of the exclusion criteria will be eligible for participation in the study. The current study was approved on 26 March 2019. The trial began on 7 May 2019, and over 251 eligible patients have been enrolled by 10 January 2020 .

After acquiring written informed consent from the patients, we will collect the following baseline data: demographic data, comorbidity, surgical methods, history of surgeries, and the main results of physical and laboratory examinations. The Pittsburgh Sleep Quality Index [28] and Mini-Mental State Examination [29] will be used to evaluate the preoperative sleep quality and cognition, respectively.

\section{Randomization, grouping, and blinding}

A statistician will complete the generation of random numbers using a computer at a ratio of 1:1 that will then be sealed in envelopes. One of the study personnel, who is not acquainted with the patients before randomization and who will not participate in the anesthesia management or postoperative follow-up of the enrolled patients, will open the envelope according to the order of the random numbers and prepare the study drugs before central venous catheterization.

The investigators who perform the data collection and postoperative follow-up, patients, anesthesiologists, surgeons, and other healthcare team members will be blinded to randomization, study drug preparation, and group allocation during the study period.

An unmasked strategy will be applied immediately if the patients experience any severe adverse event or unexpected worsening of their clinical status. In addition, the research personnel will take effective measures to ensure the safety of the participants. These situations will be documented in the case report forms (CRFs). Then, the unmasked patients will be analyzed only by the intention-to-treat method.

\section{Interventions, anesthesia, and analgesia}

None of the included patients will be permitted to receive premedication as the anticholinergic agent, such as pentylequine or scopolamine. Parameters, such as electrocardiogram, invasive blood pressure, central venous pressure, pulse oxygen saturation, end-tidal carbon dioxide, nasopharyngeal temperature, urine output, and the bispectral index (BIS), will be monitored intraoperatively.

Dexmedetomidine (Jiangsu Hengrui Medicine, Jiangsu, China) $(200 \mu \mathrm{g}, 2 \mathrm{~mL})$ and saline will be utilized as the study medicines. A dose of $0.6 \mu \mathrm{g} / \mathrm{kg}$ dexmedetomidine will be diluted in $20 \mathrm{~mL}$ saline, and $4 \mu \mathrm{g} / \mathrm{kg}$ will be diluted into $40 \mathrm{~mL}$. The loading dose will be administered as $120 \mathrm{~mL} / \mathrm{h}$ for $10 \mathrm{~min}$ via 20 - $\mathrm{mL}$ syringes after central venous catheterization, followed by continuous administration at a rate of $4 \mathrm{~mL} / \mathrm{h}(0.4 \mu \mathrm{g} / \mathrm{kg} / \mathrm{h})$ until the end of surgery. The same volume and infusion rate will be applied for patients in the saline group.

The attending anesthetists will attenuate or cease the infusion of the study drug under the following conditions: (1) severe hypotension or bradycardia that does not improve after routine therapy, (2) a worsening or new onset of atrioventricular block of degree $\geq 2$ that does not improve after routine treatment, and (3) other conditions. We will record the reason for any protocol deviations in the CRFs and analyze the data from these patients by using the intention-to-treat method but not the per-protocol analysis. We will provide the intervention drugs for free as compensation.

The intravenous medications for anesthesia induction will include midazolam, sufentanil, etomidate, and cisatracurium, and the anesthetics for sustaining anesthesia will include propofol, sufentanil, cisatracurium, and sevoflurane. Additional dexmedetomidine will be infused during surgery in the DEX group according to the study program. The BIS will be used to monitor the depth of anesthesia and maintained between 40 and 60 during surgery. The body temperature will be kept except for mild hypothermia (nasopharyngeal temperature 30$34^{\circ} \mathrm{C}$ ) during aortic clamping. Mechanical ventilation parameters during off-pump will be set to a tidal volume of $8-10 \mathrm{~mL} / \mathrm{kg}$, respiratory rate of $12-14 / \mathrm{min}$, ratio of time of inhalation, and exhalation at 1:2, and the pressure of end-tidal carbon dioxide will be kept between 35 and $45 \mathrm{mmHg}$. The inhaled gas will be a mixture of air and oxygen, and the fraction of inspired oxygen $\left(\mathrm{FiO}_{2}\right)$ will not exceed $50 \%$. If the pulse oxygen saturation has a continuously low value, and there is no response to treatment involving positive end-exhalation pressure ventilation, a high $\mathrm{FiO}_{2}$ will be administered. The hemodynamic stability of the patients will be maintained by adjusting the depth of the anesthesia and applying vasoactive drugs during surgery.

All patients will be transferred to the ICU and shifted back to general cardiac wards after several days. All participants will be equipped with patient-controlled intravenous analgesia (PCIA) pump containing $100 \mu \mathrm{g}$ sufentanil and $15 \mathrm{mg}$ dezocine in $100 \mathrm{~mL}$ saline. The background infusion rate will be $2 \mathrm{~mL} / \mathrm{h}$. The pump will deliver an additional 2-mL bolus with a lockout interval of $10 \mathrm{~min}$. Furthermore, intravenous sufentanil or dezocine or oral aminophen oxycodone (oxycodone hydrochloride $5 \mathrm{mg}$ and acetaminophen $325 \mathrm{mg}$ ) will be used to control pain after surgery, and the score of the rest numerical rating scale (NRS is a $0-10$-point pain scale tool: 0 indicates no pain and 10 indicates worst pain) is over 4 after three consecutive PCIA boluses. Propofol and/or midazolam will primarily be used for sedating the patients after surgery and maintaining the target 
sedation depth (a Richmond Agitation-Sedation Scale [RASS] score -2 to 1) [30]. The ICU physicians will perform the treatment of the circulatory system and decide the time of extubation and ICU discharge, according to routine management.

\section{Outcome assessment}

RASS is a questionnaire that evaluates the sedation status of a patient with a score ranging from -5 to +4 . A score of 0 indicates that the patient is in a calm and awake state. The higher the score, the more severe the patient's agitation. Conversely, the lower the score, the deeper the patient's sedation level [31]. The delirium will be assessed using the Confusion Assessment Method for the Intensive Care Unit (CAM-ICU) only when the RASS score is $>-3$. The CAM-ICU is an effective tool for assessing delirium, and it contains four parts: (1) sudden variation in mental status and behavior during the past $24 \mathrm{~h},(2)$ inattention, (3) disordered thinking, and (4) an altered level of consciousness. If the patient exhibits (1), (2), (3), or (4), he/she is diagnosed with delirium [32]. Additionally, three subtypes of delirium can be determined using the RASS scores: (1) hypoactive type, RASS score <0; (2) hyperactive type, RASS score > 0 ; and (3) mixed type, the hypoactive and hyperactive types occur alternately [33].

\section{Primary endpoints}

The primary endpoint is the prevalence of delirium during the first 7 days after cardiac valve surgery. The investigators, blinded to the preoperative and intraoperative information of the patients, will perform the follow-up of the primary and secondary outcomes. Delirium will be assessed only once on day 1 after surgery within $24 \mathrm{~h}$. From days 2-7 after surgery, the delirium assessment will be performed twice daily (8:00-10:00 and 18:00-20: 00 ) by recording the duration and subtypes of delirium. The occurrence of delirium during actual hospitalization will be considered for patients who are discharged or deceased within 5 days after surgery. In the case of discontinuation of the study drugs during surgery in patients due to side effects or those who undergo emergency surgery again within 7 days after initial surgery, a follow-up will proceed and intention-to-treat analysis will only be used to manage the data.

\section{Secondary endpoints}

The secondary endpoints will include the duration of mechanical ventilation after surgery, the number of days in the ICU and the length of hospital stay after surgery, the incidence of hypotension during and/or after dexmedetomidine infusion, the incidence of acute kidney injury ( $\mathrm{a} \geq 1.5$-fold increment in creatinine from the baseline value) and sudden arrhythmia during the hospital stay after surgery, and the mortality within 30 and 90 days after surgery.

\section{Safety management}

All unexpected events that are unfavorable to the prognosis of the patients throughout the entire medical process will be considered as adverse events. The time, management strategies, and consequences of all adverse events will be recorded in the CRFs. Severe adverse events refer to those that may lead to disability, deformity, or even death, and they will be reported to the Clinical Research Ethics Committee of the Chinese Academy of Medical Sciences, Fuwai Hospital, within $24 \mathrm{~h}$.

\section{Delirium}

Dexmedetomidine and/or haloperidol will be injected to control the condition. If the patient has recurrent delirium, we will consult a psychiatrist for treatment.

\section{Tachycardia}

Tachycardia is defined as a heart rate of $>100$ beats/ min. The management will include adjusting the dose and infusion rate of vasoactive agents, deepening the anesthesia, and administering the $\beta 1$-adrenoreceptor blocker intravenously.

\section{Bradycardia}

Bradycardia is defined as a heart rate of $<45$ beats $/ \mathrm{min}$. The management will involve suspending the surgical operation and adjusting the dose of the vasoactive agent or the infusion rate. If necessary, isoproterenol will be injected intravenously or a pacemaker will be used. The administration of anticholinergic drugs (penehyclidine and scopolamine) will be avoided because of their anticholinergic effect on the CNS.

\section{Hypotension}

Hypotension is defined as a systolic blood pressure $<80$ $\mathrm{mmHg}$ or a decrement of $>30 \%$ from the preoperative value. The management will include appropriate infusion, adjustment of the anesthesia depth according to BIS, and administration of vasoactive agents.

\section{Hypertension}

Hypertension is a systolic blood pressure $>160 \mathrm{mmHg}$ or an increment of $>30 \%$ from the baseline value. The management will involve adjusting the dose and infusion rate of the vasoactive agents, deepening the anesthesia, and administering a vasodilator intravenously.

\section{Arrhythmia}

Arrhythmia included frequent atrial or ventricular premature beats, supraventricular or ventricular tachycardia, and the new onset of atrial fibrillation. The 
treatment strategy will include the suspension of the operation, intravenous infusion of antiarrhythmic drugs, and cardiac cardioversion.

\section{Ventricular fibrillation or cardiac arrest}

The treatment will include external or intrathoracic heart compression, electrical defibrillation, infusion of epinephrine or other vasoactive agents, intravenous infusion of antiarrhythmic drugs, and an emergency CPB.

\section{Data management}

The original data will be recorded in the CRFs and the EpiData 3.1 software (The EpiData Association, Odense, Denmark) and maintained confidential. Two investigators will be responsible for the collection and entry of the data. Another two independent investigators will contribute to checking the accuracy of the study data. The implementation of the study and collection and entry of the data will be supervised by DSMB. The data management and statistical analysis will be performed by the Biostatistics Department of the Chinese Academy of Medical Sciences, Fuwai Hospital. Because dexmedetomidine is a commonly used medicine in clinical anesthesia, and there have been no reports of serious adverse events, we will not perform an interim analysis.

\section{Statistical analysis}

\section{Sample size calculation}

PASS 15.0 software (NCSS Corp., Kaysville, UT, USA) is used to calculate the sample size. According to a previous study, the incidence of delirium in patients following cardiac valve surgery was about $13.6 \%$ [34]. We estimated a $50 \%$ reduction in the incidence of delirium in such patients after an intraoperative dexmedetomidine infusion based on a preliminary experiment by the Chinese Academy of Medical Sciences, Fuwai Hospital. The ratio of the patients' numbers in the DEX and PLA groups is $1: 1$. Considering a $5 \%$ loss of cases, 652 patients will be required, with a power of $80 \%$ and a twosided $\alpha$ level of 0.05 .

\section{Outcomes analysis}

SPSS 22.0 software (IBM Co., Armonk, NY, USA) will be used to analyze the data. The continuous variables that have a normal distribution will be presented as the mean \pm standard deviation (SD) and compared using the independent sample $t$ test. The continuous data with an abnormal distribution will be presented as the median, interquartile range (IQR), and range and compared using the Mann-Whitney U test. Categorical variables will be expressed as the percentage and compared using Pearson's chi-square test. The Levene test or the HodgesLehmann estimator will be used for estimating the difference (and 95\% confidence interval) between the two means or medians. The Kaplan-Meier estimates will be applied for the time to event results. Post hoc subgroup analysis will also be done according to different age gaps of included patients, respectively. A two-sided $p$ value $<$ 0.05 will be considered to be a significant difference.

\section{Discussion}

This single-center, prospective, double-blinded, randomized, placebo-controlled trial will observe the effect of dexmedetomidine administration during surgery on delirium in adult patients following cardiac surgery.

A large number of adult patients undergo cardiac surgeries worldwide every year, and coronary artery bypass graft (CABG) and cardiac valve surgery account for the majority of cardiac surgeries $[35,36]$. Open heart cardiac surgery is a high-risk factor associated with POD due to the medical interventions, including $\mathrm{CPB}$ and hypothermia, during surgery [37]. Another study showed that compared to CABG, the patients undergoing cardiac valve surgery exhibited a high incidence of POD [38]. Therefore, we select patients undergoing cardiac valve surgery as subjects of the current study. Since delirium can occur in all ages of patients undergoing surgery, those included in the current study are not confined to the age group of $\geq 65$ years, but to all adults $>18$ years of age. The data will be analyzed through post hoc subgroup analysis based on different age groups.

Dexmedetomidine has been widely used as an adjuvant of general anesthesia in patients undergoing cardiac and major non-cardiac surgeries or as a sedative in severe patients in ICU owing to its pharmacological functions of sedation, analgesia, anti-inflammation, and antioxidation [39-41]. The dexmedetomidine infusion in this study will begin between the completion of central venous catheterization and the onset of surgery and be maintained until the end of surgery. It will be infused at a routine rate with the loading dose of $0.6 \mu \mathrm{g} / \mathrm{kg}$ for 10 $\mathrm{min}$, followed by $0.4 \mu \mathrm{g} / \mathrm{kg} / \mathrm{h}$ to the end of the surgery. Moreover, the potential side effects induced by dexmedetomidine loading dose, including bradycardia, hypotension, and atrioventricular heart-block, under general anesthesia, do not exert significant hemodynamic instability in the patients according to our preliminary test and completed cases. Furthermore, the data about bradycardia and hypotension during or after dexmedetomidine infusion will be recorded as secondary outcomes; we developed effective measures against these effects.

Some intra- and postoperative medical interventions are the main risk factors associated with POD, such as surgery trauma, $\mathrm{CPB}$, anesthetics, hypothermia, infusion, mechanical ventilation, and ICU environment [37, 42]. A previous study included 285 participants from $\mathrm{Li}$ et al. [23] and obtained a negative result in POD incidence 
through a loading dose of $0.6 \mu \mathrm{g} / \mathrm{kg}$ before surgery, followed by a continuous infusion rate of $0.4 \mu \mathrm{g} / \mathrm{kg} / \mathrm{h}$ to the end of surgery and $0.1 \mu \mathrm{g} / \mathrm{kg} / \mathrm{h}$ after surgery to the end of mechanical ventilation as compared to placebo. However, a prospective study with a sample size of 60 cases from Shu et al. [25] found that intraoperative infusion of dexmedetomidine (a loading dose of $1.0 \mu \mathrm{g} / \mathrm{kg}$ before anesthesia induction, followed by a continuous infusion rate of $0.5 \mu \mathrm{g} / \mathrm{kg} / \mathrm{h}$ till the end of surgery) significantly decreased the incidence of POD in patients undergoing cardiac surgery as compared to that of placebo. However, the small-scale sample size made the result of this study lack reliability. Another study displayed that simple postoperative low-dose dexmedetomidine infusion $(0.1 \mu \mathrm{g} / \mathrm{kg} / \mathrm{h})$ significantly decreased the POD incidence in patients undergoing non-cardiac surgery as compared to placebo [19]. These findings raised a question: is intraoperative dexmedetomidine infusion required to reduce the POD incidence in patients undergoing cardiac surgery? Therefore, it is necessary to perform two RCTs with large-scale sample size to address this concern based on intra- and postoperative dexmedetomidine administration, respectively.

The sample size is a rough value that varies depending on the results of the references and methods of calculation. According to a prospective screening by Järvelä et al. [34], the incidence of POD was about $13.6 \%$ in patients undergoing cardiac valve surgery. However, only a few studies explored the effect of simple intraoperative dexmedetomidine infusion on POD in patients undergoing cardiac surgery. Although the study by Shu et al. showed specific POD incidence after intraoperative dexmedetomidine infusion, it could not be used to estimate the sample size of this study because of its small-scale sample. Therefore, we calculated the sample size of this study through a preliminary test, including 100 cases, and the incidence of delirium was reduced by $50 \%$ at our Center through intraoperative dexmedetomidine infusion. Finally, 652 patients were required in this study, which is the largest sample size for investigating the effect of intraoperative dexmedetomidine infusion on POD in patients undergoing cardiac surgery. In addition, an accurate sample size could be obtained by interim analysis, but since dexmedetomidine is a routine clinical medication in our Center, we did not perform interim analysis. This could be a limitation of this study.

Currently, the most commonly used assessment scales for delirium are CAM-ICU and CAM. For patients in $\mathrm{ICU}$, the preference for CAM-ICU scale has become a consensus, while for those in general wards, CAM is preferred to assess delirium [23, 43]. However, the criteria for defining the occurrence of POD are consistent between CAM-ICU and CAM, i.e., the same diagnostic items for delirium include (1) acute onset of mental status changes or a fluctuating course, (2) inattention, (3) disorganization thinking, and (4) an altered level of consciousness. The patients are diagnosed to be delirious if both features 1 and 2 were present in addition to either feature 3 or 4 [32]. Since this study mainly focuses on the occurrence of POD, but not on the extent of delirium, we uniformly used CAM-ICU to assess the occurrence of delirium whether the patients are in ICU or general ward after surgery.

The present study has some strengths. First, it includes the largest sample size of all studies exploring the effect of dexmedetomidine on POD in patients undergoing cardiac surgeries. Thus, this large-scale randomized controlled trial may provide convincing results. Second, our hospital is a word-class center of cardiac surgery. Surgeons and anesthetists have extensive experience in the perioperative management of patients undergoing cardiac surgery. Third, we will select only those patients undergoing cardiac valve surgery to decrease the bias due to a discrepancy in the surgery types. Fourth, all investigators who will perform the follow-up will have received formal guidance about the diagnosis of delirium from a senior psychiatrist; thus, the results of the followup will be relatively reliable. Fifth, long-term endpoints will be observed. In this study, the 90-day mortality will be observed, and the long-term endpoints are crucial for evaluating the prognosis of patients.

Nevertheless, the present study had some limitations. First, it is a single-center study, which limits the dissemination of the results. Second, as some delirium patients may be missed at the follow-up time, a high incidence of delirium could occur. Third, although the RASS and CAM-ICU are widely used tools and have high sensitivity and specificity in terms of delirium diagnosis, a measurement bias is inevitable due to the subjective design of the two questionnaires. Fourth, the lack of interim analysis can lead to incorrect sample size, although that in this study is maximal.

\section{Trial status}

The current study (protocol version 3.0) was approved on 26 March 2019. The trial began on 7 May 2019, and over 200 eligible patients have been enrolled until 10 October 2019. We expect to complete the trial in August 2020.

\section{Supplementary information}

Supplementary information accompanies this paper at https://doi.org/10. 1186/s13063-020-04574-x.

Additional file 1. SPIRIT 2013 Checklist: Recommended items to address in a clinical trial protocol and related documents.

\section{Abbreviations}

POD: Postoperative delirium: CAM-ICU: The Confusion Assessment Method for the Intensive Care Unit; RASS: Richmond Agitation-Sedation Scale; 
CABG: Coronary artery bypass graft; DSMB: Data and Safety Monitoring Board; RCT: Randomized controlled trial; CPB: Cardiopulmonary bypass; CNS: Central nervous system; BIS: Bispectral index; CRFs: Case report forms

\section{Acknowledgements}

We thank Prof. Sessler (Department of Outcomes Research, Cleveland Clinic, Cleveland, $\mathrm{OH}, \mathrm{USA}$ ) for guidance on the study scheme. We were grateful for help from Zhiyi Zuo (Department of Anesthesiology, University of Virginia, Charlottesville, VA, USA) with the design of this study. We also thank Dr. Yang Wang (Department of Biostatistics, the Chinese Academy of Medical Sciences, Fuwai Hospital, China) for his help in the statistical data management. Finally, we thank all of the anesthetists for their help in conducting the trial.

\section{Authors' contributions}

$\mathrm{SY}$ and $\mathrm{F}-\mathrm{XY}$ contributed to the study design and manuscript revision. $\mathrm{H}-\mathrm{BW}$ drafted this manuscript. H-BW, ZZ, LZ, and Q-PL conducted the study. All authors read and approved the submission of the final manuscript.

\section{Funding}

The study did not receive any institutional funding, nor external, internal, or individual funding

\section{Availability of data and materials}

All authors will have full access to the final dataset data during the analysis on reasonable request

\section{Ethics approval and consent to participate}

The final study version 3.0 was approved by the Ethics Committee of the Chinese Academy of Medical Sciences, Fuwai Hospital, China on 26 March 2019 (2019-1180). Written informed consent from the patients will be obtained from each participant.

\section{Consent for publication}

Not applicable

\section{Competing interests}

The authors declare that there were no competing financial interests.

\section{Author details}

'Department of Anesthesiology, Fuwai Hospital, Chinese Academy of Medical Sciences and Peking Union Medical College, Beijing, No. 167 North Lishi Road, Xicheng District, Beijing, China. ${ }^{2}$ Department of Anesthesiology, Chongqing Traditional Chinese Medicine Hospital, Chongqing, No. 6, 7 Branch Road, Panxi, Jiangbei District, Chongqing, China.

Received: 19 March 2020 Accepted: 2 July 2020

Published online: 14 July 2020

\section{References}

1. Lipowski ZJ. Delirium (acute confusional states). JAMA. 1987;258:1789-92.

2. Munk L, Andersen G, Møller AM. Post-anaesthetic emergence delirium in adults: incidence, predictors and consequences. Acta Anaesthesiol Scand. 2016;60:1059-66

3. Sockalingam S, Parekh N, Bogoch II, Sun J, Mahtani R, Beach C, Bollegalla N, Turzanski S, Seto E, Kim J, Dulay P, Scarrow S, Bhalerao S. Delirium in the postoperative cardiac patient: a review. J Card Surg. 2005;20:560-7.

4. Gleason L, Schmitt EM, Kosar CM, Tabloski P, Saczynski JS, Robinson T, Cooper Z, Rogers SO Jr, Jones RN, Marcantonio ER, Inouye SK. Effect of delirium and other major complications on outcomes after elective surgery in older adults. JAMA Surg. 2015;150:1134-40.

5. Shurtleff V, Radosevich JJ, Patanwala AE. Comparison of Ketamine- Versus Nonketamine-Based Sedation on Delirium and Coma in the Intensive Care Unit. 2020;35:536-41.

6. O'Neal JB, Billings FT 4th, Liu X, Shotwell MS, Liang Y, Shah AS, Ehrenfeld JM, Wanderer JP, Shaw AD. Risk factors for delirium after cardiac surgery: a historical cohort study outlining the influence of cardiopulmonary bypass. Can J Anaesth. 2017:64:1129-37.

7. Leung JM, Sands LP, Newman S, Meckler G, Xie Y, Gay C, Lee K. Preoperative sleep disruption and postoperative delirium. J Clin Sleep Med. 2015;11:907-13.
8. Meier DE. Pain as a cause of agitated delirium. Arch Intern Med. 2012;172: 1130

9. Koster S, Hensens AG, van der Palen J. The long-term cognitive and functional outcomes of postoperative delirium after cardiac surgery. Ann Thorac Surg. 2009;87:1469-74.

10. Mangusan RF, Hooper V, Denslow SA, Travis L. Outcomes associated with postoperative delirium after cardiac surgery. Am J Crit Care. 2015;24:156-63.

11. Krzych LJ, Wybraniec MT, Krupka-Matuszczyk I, Skrzypek M, Bolkowska A, Wilczyński M, Bochenek AA. Detailed insight into the impact of postoperative neuropsychiatric complications on mortality in a cohort of cardiac surgery subjects: a 23,000-patient-year analysis. J Cardiothorac Vasc Anesth. 2014;28:448-57.

12. Zhang Z, Ferretti V, Güntan I, Moro A, Steinberg EA, Ye Z, Zecharia AY, Yu X, Vyssotski AL, Brickley SG, Yustos R, Pillidge ZE, Harding EC, Wisden W, Franks NP. Neuronal ensembles sufficient for recovery sleep and the sedative actions of a2 adrenergic agonists. Nat Neurosci. 2015;18:553-61.

13. Nelson S, Muzyk AJ, Bucklin MH, Brudney S, Gagliardi JP. Defining the role of dexmedetomidine in the prevention of delirium in the intensive care unit. Biomed Res Int. 2015:2015:635737.

14. Lee $C$, Lee $C H$, Lee $G$, Lee $M$, Hwang J. The effect of the timing and dose of dexmedetomidine on postoperative delirium in elderly patients after laparoscopic major non-cardiac surgery: a double blind randomized controlled study. J Clin Anesth. 2018:47:27-32.

15. Li CJ, Wang BJ, Mu DL, Hu J, Guo C, Li XY, Ma D, Wang DX. Randomized clinical trial of intraoperative dexmedetomidine to prevent delirium in the elderly undergoing major non-cardiac surgery. Br I Surg. 2020;107:e123-32.

16. Karren $E A$, King $A B$, Hughes $C G$. Dexmedetomidine for prevention of delirium in elderly patients after non-cardiac surgery. J Thorac Dis. 2016;8:E1759-62.

17. Cheng XQ, Mei B, Zuo YM, Wu H, Peng XH, Zhao Q, Liu XS, Gu E, POCD Study Group. A multicentre randomised controlled trial of the effect of intra-operative dexmedetomidine on cognitive decline after surgery. Anaesthesia. 2019:74:741-50.

18. Kim JA, Ahn HJ, Yang M, Lee SH, Jeong H, Seong BG. Intraoperative use of dexmedetomidine for the prevention of emergence agitation and postoperative delirium in thoracic surgery: a randomized-controlled trial. Can J Anaesth. 2019:66:371-9.

19. Su X, Meng ZT, Wu XH, Cui F, Li HL, Wang DX, Zhu X, Zhu SN, Maze M, Ma D. Dexmedetomidine for prevention of delirium in elderly patients after non-cardiac surgery: a randomised, double-blind, placebo-controlled trial. Lancet. 2016:388:1893-902.

20. Pereira JV, Sanjanwala RM, Mohammed MK, Le ML, Arora RC. Dexmedetomidine versus propofol sedation in reducing delirium among older adults in the ICU: a systematic review and meta-analysis. Eur J Anaesthesiol. 2020;37:121-31.

21. Burry L, Hutton B, Williamson DR, Mehta S, Adhikari NK, Cheng W, Ely EW, Egerod I, Fergusson DA, Rose L. Pharmacological interventions for the treatment of delirium in critically ill adults. Cochrane Database Syst Rev. 2019;9:CD011749

22. Ji F, Li Z, Nguyen H, Young N, Shi P, Fleming N, Liu H. Perioperative dexmedetomidine improves outcomes of cardiac surgery. Circulation. 2013; 127:1576-84.

23. Li X, Yang J, Nie XL, Zhang Y, Li XY, Li LH, Wang DX, Ma D. Impact of dexmedetomidine on the incidence of delirium in elderly patients after cardiac surgery: a randomized controlled trial. PLoS One. 2017;12:e0170757.

24. Cheng H, Li Z, Young N, Boyd D, Atkins Z, Ji F, Liu H. The effect of dexmedetomidine on outcomes of cardiac surgery in elderly patients. $J$ Cardiothorac Vasc Anesth. 2016:30:1502-8.

25. Shu A, Liu X, Wang Q, Chen X, Zhan L. Study on cerebral protective effect of dexmedetomidine during anesthesia in cardiac valve replacement surgery. Int J Clin Exp Med. 2017;10:1066-72.

26. Sheikh TA, Dar BA, Akhter N, Ahmad N. A comparative study evaluating effects of intravenous sedation by dexmedetomidine and propofol on patient hemodynamics and postoperative outcomes in cardiac surgery. Anesth Essays Res. 2018:12:555-60.

27. Chan AW, Tetzlaff JM, Gøtzsche PC, Altman DG, Mann H, Berlin JA, Dickersin K, Hróbjartsson A, Schulz KF, Parulekar WR, Krleza-Jeric K, Laupacis A, Moher D. SPIRIT 2013 explanation and elaboration: guidance for protocols of clinical trials. BMJ. 2013;346:e7586.

28. Buysse DJ, Reynolds CF 3rd, Monk TH, Berman SR, Kupfer DJ. The Pittsburgh Sleep Quality Index: a new instrument for psychiatric practice and research. Psychiatry Res. 1989;28:193-213. 
29. Folstein MF, Folstein SE, McHugh PR. "Mini-mental state". A practical method for grading the cognitive state of patients for the clinician. J Psychiatr Res. 1975;12:189-98.

30. Shehabi Y, Howe BD, Bellomo R, Arabi YM, Bailey M, Bass FE, Bin Kadiman S, McArthur CJ, Murray L, Reade MC, Seppelt IM, Takala J, Wise MP, Webb SA, ANZICS Clinical Trials Group and the SPICE III Investigators. Early sedation with dexmedetomidine in critically ill patients. N Engl J Med. 2019;380: 2506-17.

31. Sessler CN, Gosnell MS, Grap MJ, Brophy GM, O'Neal PV, Keane KA, Tesoro EP, Elswick RK. The Richmond Agitation-Sedation Scale: validity and reliability in adult intensive care unit patients. Am J Respir Crit Care Med. 2002;166:1338-44.

32. Ely EW, Inouye SK, Bernard GR, Gordon S, Francis J, May L, Truman B, Speroff T, Gautam S, Margolin R, Hart RP, Dittus R. Delirium in mechanically ventilated patients: validity and reliability of the confusion assessment method for the intensive care unit (CAM-ICU). JAMA. 2001;286:2703-10.

33. Peterson JF, Pun BT, Dittus RS, Thomason JW, Jackson JC, Shintani AK, Ely EW. Delirium and its motoric subtypes: a study of 614 critically ill patients. J Am Geriatr Soc. 2006;54:479-84.

34. Järvelä K, Porkkala H, Karlsson S, Martikainen T, Selander T, Bendel S. Postoperative delirium in cardiac surgery patients. J Cardiothorac Vasc Anesth. 2018:32:1597-602.

35. Saleh SS, Racz M, Hannan E. The effect of preoperative and hospital characteristics on costs for coronary artery bypass graft. Ann Surg. 2009; 249(2):335-41.

36. Hannan EL, Cozzens K, King SB 3rd, Walford G, Shah NR. The New York State cardiac registries: history, contributions, limitations, and lessons for future efforts to assess and publicly report healthcare outcomes. J Am Coll Cardiol. 2012;59:2309-16

37. Rudiger A, Begdeda H, Babic D, Krüger B, Seifert B, Schubert M, Spahn DR, Bettex D. Intra-operative events during cardiac surgery are risk factors for the development of delirium in the ICU. Crit Care. 2016;20:264.

38. Hudetz JA, lqbal Z, Gandhi SD, Patterson KM, Byrne AJ, Pagel PS Postoperative delirium and short-term cognitive dysfunction occur more frequently in patients undergoing valve surgery with or without coronary artery bypass graft surgery compared with coronary artery bypass graft surgery alone: results of a pilot study. J Cardiothorac Vasc Anesth. 2011;25: 811-6.

39. Weerink MAS, Struys MMRF, Hannivoort LN, Barends CRM, Absalom AR, Colin P. Clinical pharmacokinetics and pharmacodynamics of dexmedetomidine. Clin Pharmacokinet. 2017;56:893-913.

40. Bao Y, Zhu Y, He G, Ni H, Liu C, Ma L, Zhang L, Shi D. Dexmedetomidine attenuates neuroinflammation in LPS-stimulated BV2 microglia cells through upregulation of miR-340. Drug Des Devel Ther. 2019;13:3465-75.

41. Huang J, Jiang Q. Dexmedetomidine protects against neurological dysfunction in a mouse intracerebral hemorrhage model by inhibiting mitochondrial dysfunction-derived oxidative stress. J Stroke Cerebrovasc Dis. 2019;28:1281-9.

42. Smulter N, Lingehall HC, Gustafson Y, Olofsson B, Engström KG. Delirium after cardiac surgery: incidence and risk factors. Interact Cardiovasc Thorac Surg. 2013;17:790-6

43. Shi C, Jin J, Qiao L, Li T, Ma J, Ma Z. Effect of perioperative administration of dexmedetomidine on delirium after cardiac surgery in elderly patients: a double-blinded, multi-center, randomized study. Clin Interv Aging. 2019;14: $571-5$.

\section{Publisher's Note}

Springer Nature remains neutral with regard to jurisdictional claims in published maps and institutional affiliations.

Ready to submit your research? Choose BMC and benefit from:
- fast, convenient online submission
- thorough peer review by experienced researchers in your field
- rapid publication on acceptance
- support for research data, including large and complex data types
- gold Open Access which fosters wider collaboration and increased citations
- maximum visibility for your research: over 100M website views per year
At BMC, research is always in progress.
Learn more biomedcentral.com/submissions

